\title{
SOME REMARKS ON LIE FLOWS
}

\author{
M. LLABRÉS AND A. REVENTÓS
}

\begin{abstract}
The first part of this paper is concerned with geometrical and cohomological properties of Lie flows on compact manifolds. Relations between these properties and the Euler class of the flow are given.

The second part deals with 3-codimensional Lie flows. Using the classification of 3-dimensional Lie algebras we give cohomological obstructions for a compact manifold admits a Lie flow transversely modeled on a given Lie algebra.
\end{abstract}

\section{Introduction}

Let $\mathcal{F}$ be a foliation on a manifold $M$ given by an integrable subbundle $L \subset T M$. The complex of basic forms is the subcomplex $\Omega^{*}(M / \mathcal{F}) \subset \Omega^{*}(M)$ of the De Rham complex given by the forms $\alpha$ satisfiying $L_{X} \alpha=0$ and $i_{X} \alpha=0$ for all $X \in \Gamma L$. The cohomology of this complex, $H^{*}(M / \mathcal{F})$, is called the basic cohomology of the foliated manifold $(\mathrm{M} / \mathcal{F})$.

A. El Kacimi and G. Hector proved in [3], and independently V. Sergiescu in [11], that for Riemannian foliations on compact manifolds the space of cohomology $H^{*}(M / \mathcal{F})$ satisfies Poincaré duality if and only if $H^{n}(M / \mathcal{F}) \neq 0$ (where $n=\operatorname{codim} \mathcal{F}$ ). In this case $\mathcal{F}$ will be called unimodular.

In the case of Riemannian flows, it is proved by Molino-Sergiescu (cf. [8]) that $\mathcal{F}$ is unimodular if and only if it is isometric, i.e. there exists a Rjemannian metric $g$ on $M$ and a unit vector field $X$ tangent to $\mathcal{F}$ which generates a group of isometries.

The Euler class of an isometric flow $\mathcal{F}$ can then be defined by (cf. [9])

$$
e(\mathcal{F})=\left[d\left(i_{X g}\right)\right] \in H^{2}(M / \mathcal{F}) .
$$

One interesting class of Riemannian flows is constituted by Lie fiows (1dimensional foliations transversely modeled on Lie groups).

If $\mathcal{F}$ is an unimodular Lie flow transversely modeled on $G$ (a Lie group of Lie algebra $\mathcal{G}$ ) it is known (cf. [6]) that $H^{*}(\mathcal{G})$ is canonically injected in $H^{*}(M)$. Moreover, $\mathcal{F}$ is homogeneous (see $\S 1$ for definition) if and only if $e(\mathcal{F}) \in H^{2}(\mathcal{G})$.

In this paper we prosecute the study of geometrical and cohomological properties of Lie flows initiated in [6]. Some of these properties are related with the annulation of $e(\mathcal{F})$. We obtain the following results (see $\S 1$ for definitions): 
Theorem 1. Let $\mathcal{F}$ be a homogeneous Lie $\mathcal{G}$-flow on a compact manifold $M$. Then $\mathcal{F}$ is unimodular if and only if the Lie algebra $\mathcal{G}$ is unimodulat.

Theorem 2. Let $\mathcal{F}$ be an unimodular Lie flow on a compact manifold of dimension 3. Then the following conditions are equivalent:

i) $e(\mathcal{F}) \neq 0$.

ii) $\mathcal{F}$ is a contact foliation.

iii) $\mathcal{F}$ does not admit any Riemannian complementary foliation.

The second part of this paper is devoted to 3 -codimensional Lie flows. In [4] a study is made of the realization of such. flows on compact manifolds. This study is based on the classification of the 3-dimensional Lie algebras.

Using this classification we study here some particular cases. For instance

Theorem 3. Let $\mathcal{F}$ be a 3 -codimensional Lie flow on a compact manifold $M$, transversely modeled on the abelian Lie algebra $\mathcal{G}$. Then we have:

i) $\operatorname{dim} H^{1}(M ; \mathbf{R})=3$ (and the leaves of $\mathcal{F}$ are the fores of a non-trivial $\mathrm{S}^{1}$-bundle over the torus $\left.\mathrm{T}^{3}\right)$.

or

ii) $\operatorname{dim} H^{1}(M ; \mathrm{R})=4$ (and $M$ is the torus $\mathrm{T}^{4}$ ).

On the other hand it is proved in [4] that every Lie flow of codimension 3 and structural Lie algebra $\mathcal{G}$ of dimension 2 must be modeled on $\mathcal{G}_{1}, \mathcal{G}_{5}, \mathcal{G}_{7}(k \notin \mathrm{Q})$ or $\mathcal{G}_{8}$ (see the definition of $\mathcal{G}_{i}$ in $\S 1$ ). Examples for $\mathcal{G}_{1}, \mathcal{G}_{5}$ and $\mathcal{G}_{7}$ are given. Nevertheless a characterization of those algebras in the families $\mathcal{G}_{7}$ and $\mathcal{G}_{8}$ for which this situation is possible has not been given. The following result gives a cohomological restriction for the existence of such a flow on a compact manifold $M$.

Theorem 4. Let $\mathcal{F}$ be a 3-codimensional Lie flow transversely modeled on $\mathcal{G}_{7}$ or $\mathcal{G}_{8}(h \neq 0)$ and structural Lie algebra of dimension 2 . Then $\operatorname{dim} H^{1}(M ; \mathbf{R})=$ I.

Finally we relate the annulation of the Euler class with the existence of a complex structure on $M$.

Theorem 5. Let $\mathcal{F}$ be an unimodulat $\mathcal{G}$ Lise flow of codimension $S$ on a compact manifold $M$. If $\mathcal{G} \neq \mathcal{G}_{7}(k=-1)$ and $e(\mathcal{F})=0$ then $M$ is a complex manifold.

\section{Preliminaries}

Let $\mathcal{F}$ be a smooth foliation of codimension $n$ on a smooth manifold $M$ given by an integrable subbundle $L \subset T M$. We denote by $\mathcal{L}(M, \mathcal{F})$ the Lie aigebra of 
foliated vector felds, i.e. $X \in \mathcal{L}(M, \mathcal{F})$ if and only if $[X, Y] \in \Gamma L$ for all $Y \in \Gamma L$. $\Gamma L$ is an ideal of $\mathcal{L}(M, \mathcal{F})$ and the elements of $\mathcal{X}(M, \mathcal{F})=\mathcal{L}(M, \mathcal{F}) / \Gamma L$ are called basic vector fields.

If there is a family $\left\{X_{1}, \ldots, X_{n}\right\}$ of foliated vector fields on $M$ such that the corresponding family $\left\{\bar{X}_{1}, \ldots, \bar{X}_{n}\right\}$ of basic vector fields has rank $n$ everywhere the foliation is called transversely parallelizable and $\left\{\bar{X}_{1}, \ldots, \bar{X}_{n}\right\}$ a transvers parallelism. If the vector subspace $\mathcal{G}$ of $\mathcal{X}(M / \mathcal{F})$ generated by $\left\{\bar{X}_{1}, \ldots, \bar{X}_{n}\right\}$ is a Lie subalgebra, the foliation is called a Lie foliation.

We shall use the following structure theorem (cf. [7]).

Theorem. Let $\mathcal{F}$ be a transversely parallelizable foliation on a compact manifold $M$, of codimension $n$. Then

a) There is a Lie algebra $\mathcal{H}$ of dimension $y \leq n$.

b) There is a locally trivial fibration $\pi: M \rightarrow W$ with compact fibre $F$ and

$$
\operatorname{dim} W=n-g=m .
$$

c) There is a dense Lie $\mathcal{H}$-foliation on $F$ such that:

i) The fibres of $\pi$ are the adherences of the leaves of $\mathcal{F}$.

i i) The foliation induced by $\mathcal{F}$ on a fibre $F$ of $\pi$ is isomorphic to the $\mathcal{H}$-foliation on $F$.

$\mathcal{H}$ is called the structural Lie algebra of $(M, \mathcal{F}), \pi$ the basic fibration and $W$ the basic manifold.

We shall aiso use the relation between the basic cohomology and te cohomo$\log y$ of the Lie algebra $\mathcal{G}$. In particular it is proved in [6]

Theorem A. Let $\mathcal{F}$ be an unimodular Lie $\mathcal{G}$-foliation on a compact manifold $M$. Then the Lie algebra $\mathcal{G}$ is unimodular.

Theorem B. Let $\mathcal{F}$ be a Lie $\mathcal{G}$-foliation with codim $\overline{\mathcal{F}}=1$. Then $\mathcal{F}$ is unimodular if and only if $\mathcal{G}$ is unimodular and the structural Lie algebra $\mathcal{H}$ is also unimodular.

We shall be concerned with one dimensional Lie foliations, in particular with homogeneous Lie flows:

\section{Definition.}

$A$ Lie $\mathcal{G}$-flow $\mathcal{F}$ on a compact manifold $M$ is an homogeneous Lie flow if there is a Lie group $H$ and a discrete uniform Lie subgroup $H_{0}$ of $H$ such that $M=H / H_{0}$ and there is a 1-dimensional normal subgroup $K$ of $H$ such that the leaves of $\mathcal{F}$ are the orbits of the left action of $K$ on $H$. 
Theorem C. (cf. (6) Let $\mathcal{F}$ be an unimodular Lie flow with Lie algebra $\mathcal{G}$. Then $\mathcal{F}$ is homogeneous if and only if the Euler class $e(\mathcal{F}) \in H^{2}(M / \mathcal{F})$ belongs to $H^{2}(\mathcal{G})$.

Finally, we recall the clasification of the 3-dimensional Lie algebras:

i) $\mathcal{G}_{1}$, the abelian Lie algebra.

ii) $\mathcal{G}_{2}$, the Lie algebra of the Heisenberg group.

iii) $\mathcal{G}_{3}, S O(3)$

iv) $\mathcal{G}_{4}, \mathcal{S l}(2)$

v) $\mathcal{G}_{5}, \mathcal{A} \oplus \mathbf{R}$ where $\mathcal{A}$ is the affine 2-dimensional Lie algebra.

For the remainder ones we give the product

vi) $\mathcal{G}_{6},\left[e_{1}, e_{2}\right]=0,\left[e_{1}, e_{3}\right]=e_{1},\left[e_{2}, e_{3}\right]=e_{1}+e_{2}$.

vii) $\mathcal{G}_{7},\left[e_{1}, e_{2}\right]=0,\left[e_{1}, e_{3}\right]=e_{1},\left[e_{2}, e_{3}\right]=k e_{1}+e_{2}, 0 \neq k \in \mathbf{R}$.

viii) $\mathcal{G}_{8},\left[e_{1}, e_{2}\right]=0,\left[e_{1}, e_{3}\right]=e_{2},\left[e_{2}, e_{3}\right]=-e_{1}+h e_{2}, h \in \mathbf{R}, h^{2}<4$.

\section{Unimodular Lie flows}

In this section, $\mathcal{F}$ will denote a $\mathcal{G}$-Lie flow and $X$ a unit vector feld tangent to $\mathcal{F}$.

Proposition 2.1. Let $\mathcal{F}$ be a codimension $n$ Lie flow. Then $\mathcal{F}$ is unimodular if and only if there exist $n$ foliated vector fields $Y_{1}, \ldots, Y_{n}$ which define a lie parallelism and such that $\left[Y_{i}, X\right]=0$ for $i=1, \ldots, n$.

Proof:

If $\mathcal{F}$ is unimodular $X$ generates a group of isometries with respect to a Riemannian metric $g$. We choose a transvers parallelism $Y_{1}, \cdots, Y_{n}$ orthogonal to $X$. The Lie bracket $\left[X, Y_{i}\right]$ is then at the same time tangent and orthogonal to $X$, i.e. $\left[X, Y_{i}\right]=0$. Reciprocally, if there exist $Y_{1}, \ldots, Y_{n}$ correspondig to a transverse Lie parallelism with $\left[X, Y_{i}\right]=0 \forall i$. Let $\theta, \theta^{1}, \ldots, \theta^{n}$ be the dual basis of $X, Y_{1}, \ldots Y_{n}$. The above assumption is equivalent to that $d \theta$ is a basic 2-form:

$$
i_{X} d \theta\left(Y_{i}\right)=X \theta\left(Y_{i}\right)-Y_{i} \theta(X)-\theta\left[X, Y_{i}\right]=0
$$

If $\mathcal{F}$ is not unimodular, the $n$-form

$$
\omega=\theta^{1} \wedge \cdots \wedge \theta^{n}
$$

must be exact as a basic form, i.e. there exists a (n-1)-basic form $\alpha$ such that

$$
\omega=d \alpha \text {. }
$$

The volume element $\eta$ of $M$ corresponding to the given basis of 1 -forms $\theta, \theta^{1}, \ldots, \theta^{n}$ is such that

$$
\eta=\theta \wedge \omega=\theta \wedge d \alpha=d(\theta \wedge \alpha)-d \theta \wedge \alpha
$$

Observe that $d \theta \wedge \dot{\alpha}=0$ because is a $(n+1)$-basic form.

So we obtain that the volume element of $M$ is an exact form. This is not possible. 
Proposition 2.2. Let $\mathcal{F}$ be an unimodular $\mathcal{G}$-Lie flow. Then there are equivalent:

i) $e(\mathcal{F})=0$

ii) There exist foliated vector fields $Y_{1}, \ldots, Y_{n}$ correspondig to a given Lie parallelism $\bar{Y}_{1}, \ldots, \bar{Y}_{n}$ such that for the dual basis $\theta, \theta^{\sharp}, \ldots, \theta^{n}$ of $X$, $Y_{1}, \ldots, Y_{n}$ the 1 . form $\theta$ is closed.

Proof:

i) $\Rightarrow$ ii) Assume that $X$ is a Killing vector field for a Riemannian metric $g$. If $e=d \beta$, where $\beta$ is a basic 1 -form we can modificate $g$ and the parallelism in the following way:

$$
\begin{aligned}
\tilde{g} & =g-(\chi \otimes \beta+\beta \otimes \chi) \quad\left(\chi=\mathrm{i}_{X} g\right) \\
\dot{Y}_{i} & =Y_{i}+\beta\left(Y_{i}\right) X
\end{aligned}
$$

Then we have $d\left(\mathrm{i}_{X} \hat{g}\right)=0$ and $\hat{g}\left(X, \hat{Y}_{i}\right)=0$ i.e. $\mathrm{i}_{X} \hat{g}=\theta$ where $\theta, \theta^{1}, \ldots, \theta^{n}$ is the dual basis of $X, \tilde{Y}_{1}, \ldots, \tilde{Y}_{n}$. This proves $\left.i\right) \Rightarrow$ ii).

ii) $\Rightarrow$ i) We can consider that the tangent field $X$ generates a group of isometries with respect to a Riemannian metric $g$ (cf. [8]).

The one characteristic form $\chi=i x g$ can be writien

$$
\chi=\theta+\sum_{i=1}^{n} g\left(X, Y_{i}\right) \theta^{i} .
$$

All the functions $g\left(X, Y_{i}\right)$ are basic.In fact as we can choose the vector fields $Y_{2}$, corresponding to the class $\bar{Y}_{i}$ such that $\left[X, Y_{i}\right]=0$ for $i=1, \ldots, n$ and $L_{X} g=0$ we have:

$$
\begin{aligned}
0 & =L_{X} g\left(X, Y_{i}\right)=X g\left(X, Y_{i}\right)-g\left([X, X], Y_{i}\right)-g\left(X,\left[X, Y_{i}\right]\right)= \\
& =X_{i}\left(X, Y_{i}\right) .
\end{aligned}
$$

Thus the 2-form $\beta=\sum_{i=1}^{n} g\left(X, Y_{i}\right) \theta^{i}$ is a basic form. Differentiating in (1)

$$
d \chi=d \theta+d \beta=d \beta
$$

so $e(\mathcal{F})=[d \chi]=[d \beta]=0$ in $H^{2}(M / \mathcal{F})$.

Theorem 1. Let $\mathcal{F}$ be an homogeneous Lie $\mathcal{G}$-flow. Then $\mathcal{F}$ is unimodular if and only if $\mathcal{G}$ is unimodular.

Proof: 
One implication is the Theorem A.

Assume that $\mathcal{G}$ is unimodular. Let $\left\{\vec{Y}_{1}, \ldots, \bar{Y}_{n}\right\}$ be a transvers Lie parallelism of $\mathcal{F}$. Since $\mathcal{F}$ is homogeneous the Lie brackets are

$$
\begin{aligned}
& {\left[Y_{i}, Y_{j}\right]=\sum_{k=1}^{n} c_{i j}^{k} Y_{k}+a_{i j} X} \\
& {\left[Y_{i}, X\right]=b_{i} X}
\end{aligned}
$$

where all the coefficients $c_{i j}^{k}, a_{i j}, b_{i}$ are constants, for suitabie representants of the transverse parallelism.

By Proposition 2.1 it suffices to prove that $b_{i}=0$ for $i=1, \ldots, n$.

The assumption that $G$ is unimodular implies that for all $i$

$$
L_{Y_{i} \omega}=0
$$

In fact, $L_{Y_{i}} \omega$ is an $n$-basic form and

$$
L_{Y_{i}} \omega\left(Y_{1}, \ldots, Y_{n}\right)=d i_{Y_{i}} \omega\left(Y_{1}, \ldots, Y_{n}\right)=0
$$

because the differential of every $(n-1)$-form on an unimodular Lie algebra $\mathcal{G}$ is zero.

Thus

$$
L_{\Upsilon_{i}} \omega \wedge \theta=0
$$

but

$$
0=L_{Y_{i}} \omega \wedge \theta=L_{Y_{i}}(\omega \wedge \theta)-\omega \wedge L_{Y_{i}} \theta=d i_{Y_{i}}(\eta)-\omega \wedge b_{i} \theta
$$

Then

$$
b_{i} \eta=d i_{Y_{i}} \eta
$$

If $b_{i}$ was not zero then $\eta$ will be exact. This is not possible because $\eta$ is a volume element on the compact manifold $M$.

\section{Contact flows and Euler class}

Recall that a contact flow $\mathcal{F}$ is a flow generated by a vector field $X$ associated to a contact form $\theta$, i.e.

$$
i_{X} \theta=1, i_{X} d \theta=0 \text { and } \theta \wedge(d \theta)^{n} \neq 0 \quad \text { everywhere, }
$$

with $n=\operatorname{codim} \mathcal{F}$.

Saralegui proves in ([9]) that a contact flow $\mathcal{F}$ is unimodular. The results in the above paragraph enable us to give a shorter proof of this fact in the case of Lie flows. 
Proposition 3.1. If $\mathcal{F}$ is a contact Lie flow, then $\mathcal{F}$ is unimodular.

Proof: Let $\theta$ be the contact form associated to $X$.

Ussing the same notation as in the proof of Theorem 1 we have

$$
d \theta=\sum_{i=1}^{n} b_{i} \theta \wedge \theta^{i}+\sum_{i j} a_{i j} \theta^{i} \wedge \theta^{j} .
$$

Assume that $\mathcal{F}$ is not unimodular, then there exists $b_{i} \neq 0$ (cf. proposition 2.1). This is not possible because

$$
i_{X} d \theta=\sum_{i=1}^{n} b_{i} \theta^{i} \neq 0
$$

Definition 3.2. A foliation $\mathcal{F}^{\perp}$ of codimension $n$ transverse to $\mathcal{F}$ is called complementary Riemannian foliation of $\mathcal{F}$ if there exists a Riemannian metric on $M$ such that is bundle-like with respect the two foliations $\mathcal{F} i \mathcal{F}^{\perp}$.

Proposition 3.3. Let $\mathcal{F}$ be an unimodular Lie flow on a compact manifold. Then there are equivalent:

i) $e(\mathcal{F})=0$.

ii) There exists a complementary Riemannian foliation of $\mathcal{F}$.

Proof:

i) $\Rightarrow$ ii). If $e(\mathcal{F})=0$ one can find a Lie parallelism $X, Y_{1}, \ldots, Y_{n}$ such that in the dual basis $\theta, \theta^{\mathrm{t}}, \ldots, \theta^{\mathrm{n}}$ the differential $d \theta$ is zero.

Since $\mathcal{F}$ is unimodular the $b_{i}$ coefficients are zero and we have

$$
d \theta=\sum_{i, j} a_{i j} \theta^{j} \wedge \theta^{j},
$$

then $d \theta=0$ implies $a_{i j}=0$ for all $i, j=1, \ldots, n$.

This condition means that $Y_{1}, \ldots, Y_{n}$ define a foliation transvers to $\mathcal{F}$.

Now, we have to find a $g$ such that is bundle-like with respect the two foliations.

Let $g$ be a metric for which $X$ is isometric and $Y_{\mathrm{i}}$ are ortogonal to $X$. It suffices to prove that

$$
\begin{aligned}
X g\left(Y_{i}, Y_{j}\right) & =g\left(\left[X, Y_{i}\right], Y_{j}\right)+g\left(Y_{i},\left[X, Y_{j}\right]\right) \quad \forall i, j . \\
Y_{i} g(f X, h X) & =g\left(\left[Y_{i}, f X\right], h X\right)+g\left(f X,\left[Y_{i}, h X\right]\right) \quad \forall i .
\end{aligned}
$$

The condition (1) is true because $X$ is Killing; (2) is immediat. 
ii) $\Rightarrow$ i). If $\mathcal{F}$ admits a complementary Riemannian foliation, then there exists a Lie parallelism $\left\{\tilde{\tilde{Y}}_{1}, \ldots, \overrightarrow{\tilde{Y}}_{n}\right\}$ such that the distribution $\tilde{Y}_{1}, \ldots, \tilde{Y}_{n}$ is integrable for a suitable representants of $\left\{\bar{Y}_{i}\right\}$ de $\left\{\overline{\tilde{Y}}_{i}\right\}$.

To see this, let $\mathcal{F}^{\perp}$ be the complementary Riemannian foliation.

With the metric $g$ we project over the $Z_{i}$-plane the vector fields $Y_{i}$, corresponding to the given transverse Lie parallelism, and we obtain the decomposition

$$
Y_{i}=Y_{i}^{t}+Y_{i}^{N}=Y_{i}^{\ell}+\lambda_{i} X
$$

where $\lambda$ is a function.

The vector fields $Y_{i}^{t}$ are foliated and independent:

$$
\begin{gathered}
{\left[Y_{i}^{t}, X\right]=\left[Y_{i}-\lambda_{i} X, X\right]=\left[Y_{i}, X\right]+X\left(\lambda_{i}\right) X \in T \mathcal{F}} \\
0=\sum_{i=1}^{n} \mu_{i} Y_{i}^{\ell}=\sum_{i=1}^{n} \mu_{i}\left(Y_{1}-\lambda_{2} X\right) \Longrightarrow 0=\sum_{i=1}^{n} \mu_{i} Y_{i} \Longrightarrow \mu_{i}=0 \quad \forall i .
\end{gathered}
$$

Then $Y_{i}^{t}$ define a distribution which is clearly integrable by construction.

We shall prove that the transverse parallelisn given by these foliated vector felds is a Lie parallelism:

$$
\begin{aligned}
{\left[Y_{i}, Y_{j}\right]=\sum_{k=1}^{n} c_{i j}^{k} Y_{k} } & \Longrightarrow\left[Y_{i}^{t}+\lambda_{i} X, Y_{j}^{t}+\lambda_{j} X\right]=\sum_{k=1}^{n} c_{i j}^{k} Y_{k}^{t}+\lambda_{k} X \Longrightarrow \\
& \Longrightarrow\left[Y_{i}^{t}, Y_{j}^{t}\right]+T \mathcal{F}=\sum_{k=1}^{n} c_{i j}^{k} Y_{k}^{t}+T \mathcal{F} .
\end{aligned}
$$

So

$$
\left\{\bar{Y}_{1}^{t}, \ldots, \bar{Y}_{n}^{t}\right\}
$$

is a Lie parallelism.

Since in $\left[Y_{i}^{t}, Y_{j}^{t}\right]$ there is no part tangent to $\mathcal{F}$, we have

$$
\left[Y_{2}^{t}, Y_{j}^{-t}\right]=\sum_{k=1}^{n} c_{i j}^{k} Y_{k}^{t} .
$$

Let $\theta, \theta^{1}, \ldots, \theta^{n}$ the dual basis of $X, Y_{1}^{t}, \ldots, Y_{n}^{t}$, then

$$
d \theta=0
$$

This is equivalent to $e(\mathcal{F})=0$ (cf. Proposition 2.1 ).

The following result relates the annulation of the Euler class with the existence of a contact Lie flow. 
Proposition 3.4. Let $\mathcal{F}$ be a contact Lie flow. Then the Euler class e(F) is not zero.

\section{Proof:}

Assume that $e(\mathcal{F})=0$, then there exists a transverse Lie parallelism such that $d \theta=0$; this implies that the corresponding coefficients $a_{i j}$ are all 0 .

Let $\alpha$ be the contact form associated to $X$. In this case $d \alpha$ is a 2-basic form. But

$$
\begin{aligned}
d \alpha\left(Y_{i}, Y_{j}\right) & =Y_{i} \alpha\left(Y_{j}\right)-Y_{j} \alpha\left(Y_{i}\right)-\alpha\left(\left[Y_{i}, Y_{j}\right]\right)= \\
& =-\alpha\left(\sum_{k=1}^{n} c_{i j}^{k} Y_{k}\right)=0 .
\end{aligned}
$$

Then $d \alpha=0$ is not a contact form, this is a contradiction.

Theorem 2. Let $\mathcal{F}$ an unimodular Lie flow on a compect manifold of dimension 3 . Then there are equivalent:

i) $e(\mathcal{F}) \neq 0$.

ii) $\mathcal{F}$ is a contact flow.

i3i) $\mathcal{F}$ does not admit any complementary Riemannian foliation.

Proof:

Since $i$ ) $\Leftrightarrow i i i$ ) $i$ ii) $\Rightarrow i$ ) are true for arbitrary dimension of $M$ (cf. Proposition 3.3 and 3.4 ), we have only to prove $i i i$ ) $\Rightarrow$ ii).

Assume that $\mathcal{F}$ does not admit any complementary Riemannian foliation then

$$
\left[Y_{1}, Y_{2}\right]=c_{12}^{1} Y_{1}+c_{12}^{2} Y_{2}+a_{12} X
$$

with $a_{12} \neq 0$.

The differential form $\theta$ is a contact form:

$$
d \theta=b_{3} \theta \wedge \theta^{1}+b_{2} \theta \wedge \theta^{2}+a_{12} \theta^{1} \wedge \theta^{2}
$$

so,

$$
\theta \wedge d \theta=a_{12} \theta \wedge \theta^{1} \wedge \theta^{2} \neq 0
$$

\section{Lie flows of codimension 3}

The existence of a Lie flow on a compact manifold $M$ is a very strong condition. The structure of the transverse Lie algebra determines in general many properties of $M$. As a first step in the study of this relations we restrict our attention to Lie fows of codimension 3 . In this manner we can use the classification of the 3-dimensional Lie algebras to study separately each case.

For instance, if the Lie fow is modeled on the abelian algebra we have: 
Theorem 3. Let $\mathcal{F}$ be a Lie flow of codimension 9 on a compact manifold $M$, transversely modeled on the abelian algebra $\mathcal{G}$. Then we have:

i) $\operatorname{dim} H^{1}(M ; \mathbf{R})=3$ (and the leaves of $\mathcal{F}$ are the fibres of a non-irivial $S^{1}$-bundle over the torus $T^{3}$ ).

or

ii) $\operatorname{dim} H^{1}(M ; R)=4$ (and $M$ is the torus $T^{4}$ )

Proof: Since $\mathcal{F}$ is a Lie foliation we have the holonomy representation $h: \Pi_{1}(M) \longrightarrow h\left(\Pi_{1}(M)\right)=\Gamma \subset G$, (cf.[2]) where $G$ is, in this case, the connected simply connected group associated to the abelian Lie algebra $\mathcal{G}$, i.e. $G=\mathbf{R}^{3}$ with the standard addition. $\Gamma$ is the holonony group of $\mathcal{F}$.

As $h\left(x y x^{-1} y^{-1}\right)=0, h$ induces $\tilde{h}: H^{1}(M ; Z) \longrightarrow \Gamma$.

On the other hand, the generalized Gysin sequence of a Riemannian flow, introduced by $\mathrm{G}$. Hector in [5], gives

$$
0 \longrightarrow H^{1}(M / \mathcal{F}) \longrightarrow H^{1}(M ; \mathbb{R}) \stackrel{f}{\rightarrow} H^{3}(M / \mathcal{F}) \stackrel{g}{\longrightarrow} H^{2}(M / \mathcal{F})
$$

Since $H^{3}(M / \mathcal{F})=0$ or $\mathbf{R}\left(c \hat{f}\right.$ [1]), we have $\operatorname{dim} H^{1}(M ; \mathbf{R})=\operatorname{dim} H^{1}(M / \mathcal{F})$ (if $\operatorname{im} f=0$ ) or $\operatorname{dim} H^{1}(M ; R)=\operatorname{dim} H^{1}(M / \mathcal{F})+1($ if im $f \neq 0$ ). But the basic cohomology $H^{*}(M / \mathcal{F})$ coincides with the cohomology $H^{*}(\mathcal{G})$ of the Lie algebra $\mathcal{G}$ when $\bar{\Gamma}$ is a normal subgroup of $G$ (cf.[6]). In our case, as $G$ is abelian this condition is trivially fulfilled and we have $H^{1}(M / \mathcal{F})=H^{2}(\mathcal{G})=\mathrm{R}^{3}$. This implies $\operatorname{dim} H^{1}(M ; \mathbf{R})=3$ or 4 . We study separately these two cases.

i) If $\operatorname{din} H^{1}(M ; \mathbb{R})=3, H^{1}(M ; \mathbb{Z})=\mathbb{Z}^{3}+T$, where $T$ is the torsion part of $H^{1}(M ; Z)$. Then

$$
\begin{aligned}
& \Gamma=\hat{h}\left(\Pi_{1}(M)\right)=\tilde{h}\left(\Pi_{1}(M) /<x y x^{-1} y^{-1}>\right)=\tilde{h}\left(H^{1}(M ; Z)\right)= \\
& =\tilde{h}\left(\mathbf{Z}^{3}+T\right)=\tilde{h}\left(Z^{3}\right), \text { i.e. } \Gamma \text { is generated by } \tilde{h}(1,0,0), \tilde{h}(0,1,0) \text { and } \tilde{h}(0,0,1) .
\end{aligned}
$$

As $R^{3} / \bar{\Gamma}=M / \overline{\mathcal{F}}$ is compact, the vectors $e_{1}=\tilde{h}(1,0,0), e_{2}=\tilde{h}(0,1,0)$ and $e_{3}=\tilde{h}(0,0,1)$ are linearly independent. So $\Gamma$ is a discret subgroup of $\mathbf{R}^{3}$. In particular $\mathcal{F}$ is a compact foliation and the basic manifold is $T^{3}$.

ii) The case $\operatorname{dim} H^{1}(M ; \mathbf{R})=4$ occurs only when $H^{3}(M / \mathcal{F})=\mathbf{R}$, i.e. $\mathcal{F}$ is an unimodular Lie fow. For these flows the map $g$ in the Gysin sequence is the multiplication by the Euler class $e(\mathcal{F})$. As $f$ is exhaustive, $e(\mathcal{F})$ must be zero. This implies (cf.[6]) that $\mathcal{F}$ is an homogeneous Lie flow for the Lie algebra $\mathcal{G}+\boldsymbol{R}$, that is $M=H / H_{0}$ where $H$ is the connected simply connected group associated to the Lie algebra $\mathcal{G}+\mathbb{R}$ and $H_{0}$ is a discret subgroup of $H$. As $\mathcal{G}$ is abelian, $M=T^{4}$ as stated.

In [2] it is given one example of a Lie flow of codimension 3 transversely modeled on $\mathcal{G}_{4}$ and structural Lie algebra of dimension 1 . This example is not unimodular, i.e. $H^{3}(M / \mathcal{F})=0$. In fact every example of this situation has this property. 
Proposition 4.1. Let $\mathcal{F}$ a $\mathcal{G}_{4}$. Lie flow of codimension 3 with $\mathcal{G}=S l(2)$ and structural Lie algebra $\mathcal{K}$ of dimension 1 . Then $\mathcal{F}$ is not unimodular.

Proof:

The assumption that $\mathcal{F}$ is unimodular implies that the transverse central sheaf admits a global trivialization. That is we can find a global foliated vector field $Y$ such that $\bar{Y}$ is in the center of $l(M, \mathcal{F})$.

Let $Y_{1}, Y_{2}, Y_{3}$ be the foliated vector fields such that their Lie bracket is

$$
\left[Y_{1}, Y_{2}\right]=Y_{3},\left[Y_{2}, Y_{3}\right]=Y_{1},\left[Y_{3}, Y_{1}\right]=Y_{2}
$$

There exist basic functions $f_{1}, f_{2}, f_{3}$ and a function $f$ such that

$$
Y=f_{1} Y_{1}+f_{2} Y_{2}+f_{3} Y_{3}+f X
$$

From the condition $\left[Y_{i}, Y^{\prime}\right] \in T \mathcal{F}$ wc obtain the following systems of equations:

$$
\left\{\begin{array} { l } 
{ Y _ { 1 } ( f _ { 1 } ) = 0 } \\
{ Y _ { 1 } ( f _ { 2 } ) = f _ { 3 } } \\
{ Y _ { 1 } ( f _ { 3 } ) = - f _ { 2 } }
\end{array} \quad \left\{\begin{array} { l } 
{ i = 2 } \\
{ Y _ { 2 } ( f _ { 1 } ) = f _ { 3 } } \\
{ Y _ { 2 } ( f _ { 2 } ) = 0 }
\end{array} \quad \left\{\begin{array}{l}
Y_{3}\left(f_{1}\right)=-f_{2} \\
Y_{3}\left(f_{2}\right)=-f_{1} \\
Y_{3}\left(f_{3}\right)=0
\end{array}\right.\right.\right.
$$

Derivating the system $i$ by the vector field $Y_{i}$ we obtain

$$
\left\{\begin{array} { l } 
{ Y _ { 1 } Y _ { 1 } ( f _ { 2 } ) = - f _ { 2 } } \\
{ Y _ { 1 } Y _ { 1 } ( f _ { 3 } ) = - f _ { 3 } }
\end{array} \quad \left\{\begin{array} { l } 
{ Y _ { 2 } Y _ { 2 } ( f _ { 1 } ) = f _ { 1 } } \\
{ Y _ { 2 } Y _ { 2 } ( f _ { 3 } ) = f _ { 3 } }
\end{array} \quad \left\{\begin{array}{l}
Y_{3} Y_{3}\left(f_{1}\right)=f_{1} \\
Y_{3} Y_{3}\left(f_{2}\right)=f_{2}
\end{array}\right.\right.\right.
$$

Since $M$ is compact the function $f_{1}$ will take maximum and minimum value Let $p$ be the point where $f_{1}$ takes the maximum value, and let $\varphi_{t}$ be the integral curve of $Y_{3}$ passing through $p$.

The condition $Y_{3}\left(f_{1}\right)=-f_{2}$ implies that $f_{2}(p)=0$.

As the manifold $M$ is compact, these vector fields are complet and the restriction of $f_{2}$ to the integral curve of $Y_{3}$ passing through $p$ gives the one variable differential equation:

$$
x^{\prime \prime}=x
$$

with initials conditions $x_{0}=f_{2}(p)=0, x_{0}^{\prime}=I_{3}\left(f_{2}\right)=-f_{1}(p)$.

If $f_{1}(p) \neq 0$ then the solution of the equation is not bounded, but $f_{2}$ must be bounded because it is defined on a compact manifold.

Analogously, if $q$ is a point where the function $f_{1}$ takes the minimum value then $f_{1}(q)=0$.

Hence, $f_{1}$ is constant equal zero. This means that $f_{2}=Y_{3}\left(f_{1}\right)=0$ i $f_{3}=$ $Y_{2}\left(f_{1}\right)=0$.

Then the vector field $Y$ is tangent to $\mathcal{F}$ and it can not give a trivialization of the transverse central sheaf, which is a contradiction.

If the flow is modeled on $\mathcal{G}_{7}$ or $\mathcal{G}_{8}$ we have: 
Theorem 4. Let $\mathcal{F}$ be a Lie flow of codimension 9 on a compact manifold $M$, transversely modeled on $\mathcal{G}_{7}$ or $\mathcal{G}_{8}(h \neq 0)$ and structural Lie algebra of dimension 2. Then $\operatorname{dim} H^{1}(M ; R)=1$.

Proof: It is easy to see that every 2-dimensional abelian subalgebra of $\mathcal{G}_{7}$ or $\mathcal{G}_{8}$ is an ideal. It follows from this (cf.[6]) that for every Lie flow on a compact manifold $M$ transversaly modeled on $\mathcal{G}_{7}$ or $\mathcal{G}_{8}$ there is a transverse parallelism $Y_{1}, Y_{2}, Y_{3}$ on $M$ such that

$$
\begin{gathered}
{\left[\bar{Y}_{1}, \bar{Y}_{2}\right]=0,\left[\bar{Y}_{1}, \bar{Y}_{3}\right]=\bar{Y}_{1},\left[\bar{Y}_{2}, \bar{Y}_{3}\right]=k \bar{Y}_{2} \text { for } \mathcal{G}_{7} \text { and }\left[\bar{Y}_{1}, \bar{Y}_{2}\right]=0,\left[\bar{Y}_{\bar{I}}, \bar{Y}_{3}\right]=\bar{Y}_{2},} \\
{\left[\bar{Y}_{2}, \bar{Y}_{3}\right]=-\bar{Y}_{1}+h \bar{Y}_{2} \text { for } \mathcal{G}_{8}, \text { with } Y_{1} \text { and } Y_{2} \text { tangent to } \overline{\mathcal{F}} \text { everywhere }}
\end{gathered}
$$

On the other hand every basic closed 1-form $\theta$ can be written as

$$
\theta=f \theta^{2}+g \theta^{2}+h \theta^{3}
$$

where $\theta^{0}, \theta^{1}, \theta^{2}, \theta^{3}$ is the dual basis of $X, Y_{1}, Y_{2}, Y_{3}$ ( $X$ the generator of the flow) and $f, g, h$ are basic functions.

Next observe that since $Y_{1}(f)=Y_{2}(f)=0$ (and the same for $g$ and $h$ ) we have

$$
\begin{aligned}
& 0=d \theta\left(Y_{1}, Y_{3}\right)=-Y_{3}(f)-f-g \\
& 0=d \theta\left(Y_{2}, Y_{3}\right)=-Y_{3}(g)-f-g
\end{aligned}
$$

This system of differential equations can be interpreted now as a system on the basic manifold $S^{1}$.

For $\mathcal{G}_{7}$ we have

$$
\begin{gathered}
f^{\prime}=-f \\
g^{\prime}=-k g
\end{gathered}
$$

and for $\mathcal{G}_{8}$

$$
\begin{gathered}
f^{\prime}=-g \\
g^{\prime}=f-h g
\end{gathered}
$$

Thus in both cases we obtain $f=g=0$. (If $h=0$ the second system has non trivial periodic solutions, but this case is not considered here).

Thus every basic closed $1-$ form $\theta$ can be expressed as $\theta=h \cdot \theta^{3}$. As $\theta^{3}$ is projectable on the basic manifold $S^{1}$ we have $h \cdot \theta^{3}=\theta^{3}+d \alpha$ where $\alpha$ is a basic function on $M$. Thus $\operatorname{dim} H^{1}(M / \mathcal{F})=\operatorname{dim} H^{1}\left(S^{1} ; \mathbf{R}\right)=1$.

On the other hand, it is proved in [4] that in the hypothesis of this Theorem, $k \notin Q$. In particular $k \neq-1$ and hence $\mathcal{G}_{7}$ and $\mathcal{G}_{8}(h \neq 0)$ are not unimodular. This implies that the flow $\mathcal{F}$ is not unimodular (cf. $[6]$ ), i.e $H^{3}(M / \mathcal{F})=0$. Using now the generalized Gysin sequence

$$
0 \longrightarrow H^{1}(M / \mathcal{F}) \longrightarrow H^{1}(M ; \mathbf{R}) \longrightarrow H^{3}(M / \mathcal{F})
$$


we obtain $\operatorname{dim} H^{1}(M ; \mathbf{R})=\operatorname{dim} H^{1}(M / \mathcal{F})=1$.

Finally we relate the annulation of the Euler class with the existence of a natural complex structure on $M$. Recall that a unimodular Lie flow must be transversely modeled on a unimodular Lie algebra. As we are in codimension 3 the only possibilities are $\mathcal{G}_{1}, \mathcal{G}_{2}, \mathcal{G}_{3}, \mathcal{G}_{4}, \mathcal{G}_{7}(k=-1), \mathcal{G}_{8}(h=0)$.

Theorem 5. Let $\mathcal{F}$ be an unimodalar $\mathcal{G}$ Lie flow of codimension 9 on $a$ compact manifold $M$. If $\mathcal{G} \neq \mathcal{G}_{7}(k=-1)$ and $e(\mathcal{F})=0, M$ is a complex manifold.

Proof: We know (cf. Proposition 2.2) that there are independent vector fields $X, Y_{1}, Y_{2}, Y_{3}$ such that $X$ is tangent to $\mathcal{F},\left\{Y_{1}, Y_{2}, Y_{3}\right\}$ is a tranvers parallelism on $M$ with $\left[X, Y_{1}\right]=0$ and $\left[Y_{i}, Y_{j}\right]=c_{i j}^{k} Y_{k}$, where $c_{i j}^{k}$ are the structure constants of $\mathcal{G}$.

We define an almost complex structure $J$ on $M$ by $J X=Y_{1}, J Y_{1}=-X$, $J Y_{2}=Y_{3}, J Y_{3}=-Y_{2}$

Obviously $J^{2}=-$ id.

The torsion of $J$ is given by

$$
N(Y, Z)=[J Y, J Z]-[Y, Z]-J[J Y, Z]-J[Y, J Z]
$$

Computing it on $X, Y_{1}, Y_{2}, Y_{3}$ we obtain

$$
\begin{aligned}
N\left(X, Y_{1}\right)= & {\left[J X, J Y_{1}\right]-\left[X, Y_{1}\right]-J\left[J X, Y_{1}\right]-} \\
& -J\left[X, J Y_{1}\right] \\
= & {\left[Y_{1},-X\right]-\left[X, Y_{1}\right]-J\left[Y_{1}, Y_{1}\right]-} \\
& -J[X,-X]=0 . \\
N\left(X, Y_{2}\right)= & {\left[Y_{1}, Y_{3}\right]-\left\lfloor X, Y_{2}\right]-J\left[Y_{1}, Y_{2}\right] } \\
& -J\left[X, Y_{3}\right] \\
& =\left[Y_{1}, Y_{3}\right]-J\left[Y_{1}, Y_{2}\right]
\end{aligned}
$$

Analogously

$$
\begin{aligned}
& N\left(X, Y_{3}\right)=-J\left(\left[Y_{1}, Y_{3}\right]-J\left[Y_{1}, Y_{2}\right]\right) \\
& N\left(Y_{1}, Y_{2}\right)=-J\left(\left[Y_{1}, Y_{3}\right]-J\left[Y_{1}, Y_{2}\right]\right) \\
& N\left(Y_{1}, Y_{3}\right)=-\left\{Y_{1}, Y_{3}\right]+J\left[Y_{1}, Y_{2}\right\} \\
& N\left(Y_{2}, Y_{3}\right)=0
\end{aligned}
$$

Hence $N=0$ if and only if $\left[Y_{1}, Y_{3}^{-}\right]=J\left[Y_{1}, Y_{2}\right]$. But for $\mathcal{G}_{1}$ and $\mathcal{G}_{2}$ we have $\left[Y_{1}, Y_{2}\right]=\left[Y_{1}, Y_{3}\right]=0$ and for $\mathcal{G}_{3}$ and $\mathcal{G}_{4}$ we have $\left[Y_{1}, Y_{2}\right]=Y_{3},\left[Y_{1}, Y_{3}\right]=-Y_{2}$. For $\mathcal{G}_{8}(h=0)$ one must modify a little the definition of $J$. In fact, if we 
put $J X=Y_{3}, J Y_{3}=-X, J Y_{2}=Y_{1}, J Y_{1}=-Y_{3}$ the integrability condition becomes $\left[Y_{3}, Y_{1}\right]=-Y_{2}$ and $\left[Y_{3}, Y_{2}\right]=Y_{1}$ which is certainly satisfied. So in all these cases (the only ones considered in the Theorem) $J$ is integrable, i.e. $M$ is a complex manifold.

Remark 1. As $H^{2}\left(\mathcal{G}_{3}\right)=H^{2}\left(\mathcal{G}_{4}\right)=0$ the condition $e(\mathcal{F})=0$ is always satisfied in these cases if $\mathcal{F}$ is homogeneus.

Remark 2. If $\mathcal{G}=\mathcal{G}_{i}, M$ is a complex torus. In fact in this case for each vector field $Y$ on $M$ we have

$$
[Z, J Y]=J[Z, Y] \text { where } Z=X, Y_{1}, Y_{2} \text {, or } Y_{3}
$$

i.e. $Z$ is an infinitesimal automorphism of the complex structure. Hence

$$
X-i J X=X-i Y_{1}
$$

and

$$
Y_{2}-i J Y_{2}=Y_{2}-i Y_{3}
$$

are holomorphic vector fields on $M$. As they are $C$-independent in each point they give a complex parallelism on $M$ and $M$ is a complex torus. 


\section{References}

1. A. El KaClmi-A laOUi, G. HeCtor, Décomposition de Hodge basique pour un feuilletage riemannien, Ann. Inst. Fourier 36-2 (1986), 207-227.

2. A. EL KACIMI-ALAOUI, M. NICOLAU, Espaces homogenes moyennables des feuilletages de Lie, $P_{u b}$. IRMA Lille 12 n.7. (1988).

3. E. FEDIDA, Sur les feuilietages de Lie, C.R. Acad. Sci. (1971), 999-1002, Paris 272.

4. E. Gallego, A. Reventós, Lie flows of codimension 3. To appear

5. G. HECTOR, Cohomologies transversales des feuilletages Riemanniens, Publicacions Centre de Recerca Matemàtica. Universitat Autónoma de Barcelona.

6. M. Llabres, A. Reventós, Unimodular Lie foliations, Annales Fac. Sci. (1988), Toulouse IV (5 serie).

7. P. Molino, Géométrie globale des feuilletages riemanniens, Proc. Kon. Nederl. Akad., ser. A.1, 85 (1982), 45-76.

8. P. Molino, V. Sergiescu, Deux remarques sur les flots riemanniens, Manuscrita Math. 51 no 1-3 (1985), 145-161.

9. M. SaRAlegui, The Euler class for flows of isometries, In Research Notes in Mathematics 131.

10. V. SERGIESCU, Cohomologie basique et dualité des feuilletages riemanniens, Ann. Inst. Fourier 35-3 (1985), 137-158.

M. LLabrés: Departament de Matematiques

Universitat Autònoma de Barcelona

Bellaterra

Barcelona, SPAIN

A. Reventós: Departament de Matemàtiques

Universitat Autónoma de Barcelona

Bellaterra

Barcelona, SPAIN

Rebut el 20 de Maig de 1989 